\title{
CARACTERIZAÇÃO REOLÓGICA DA MUCILAGEM DE Cereus hildmaniannus
}

\author{
E. NIEHUES ${ }^{1}$, V. C. COLONETTI ${ }^{1}$, S. R. ZOLDAN ${ }^{2}$, M. G. N. QUADRI ${ }^{1}$ \\ ${ }^{1}$ Universidade Federal de Santa Catarina, Departamento de Engenharia Química e Engenharia de \\ Alimentos \\ ${ }^{2}$ EPAGRI, Campos Novos, SC \\ E-mail para contato: mara.quadri@ufsc.br
}

\begin{abstract}
RESUMO - O cacto Cereus hildmaniannus K. Schum cresce em solo pedregoso com escassez de água no meio oeste catarinense. Seus frutos e cladódios possuem polpa mucilaginosa com propriedades desconhecidas. Esta mucilagem é um carboidrato complexo com alta capacidade de absorção de água que pode ser utilizada como um hidrocoloide na indústria alimentícia. Este trabalho tem como objetivo caracterizar a mucilagem extraída dos frutos e cladódios através da viscosidade intrínseca. A viscosidade das soluções aquosas de mucilagem, em concentrações de 0,1 a $0,5 \mathrm{~g} \cdot \mathrm{L}^{-1}$, foi medida usando um viscosímetro capilar Cannon-Fenske, 75 . Os resultados mostraram valores de viscosidade intrínseca e concentração crítica de overlap próximos de $657 \mathrm{~mL} \cdot \mathrm{g}^{-1}$ e 1,52 , respectivamente. As constantes de Huggins e Kraemer foram 0,44 e -0,0001. Estes valores indicam que a água foi um bom solvente para a mucilagem nestas concentrações, isto é, a cadeia polimérica está solvatada, indicando boa interação entre polímero-solvente.
\end{abstract}

\section{INTRODUÇÃO}

A família Cactaceae é representada por plantas que possuem polpa mucilaginosa em seus frutos e cladódios. O cacto Cereus hildmaniannus K. Schum é uma espécie pouco estudada que cresce naturalmente em solo pedregoso e com ausência de água, condição encontrada em Zortéa, região centro-oeste de Santa Catarina.

A mucilagem é um açúcar complexo que faz parte das fibras alimentares (Sáenz et al., 2004). A estas fibras estão associados efeitos fisiológicos positivos no corpo humano como a diminuição do colesterol no sangue, controle da glicose em diabéticos e redução do risco de alguns tipos de câncer (Periago et al., 1993). Em função da sua elevada capacidade de absorção de água, a mucilagem pode ser considerada como um potencial hidrocoloide tanto para o uso na indústria alimentícia como em outras. De acordo com Jani et al. (2009), a mucilagem possui algumas características e vantagens por serem materiais obtidos de plantas: biodegradáveis, baixo custo, provenientes de fontes comestíveis, disponibilidade local e processamento amigável ao meio ambiente. $\mathrm{O}$ alto potencial de aplicações destes biopolímeros, com propriedades estruturais, físico-químicas e funcionais amplas tem estimulado a exploração e pesquisa de novos polissacarídeos (Geresh et al., 2000). 
Polissacarídeos são utilizados na indústria de alimentos para modificar as propriedades reológicas de alguns produtos (Lai et al., 2000). Extratos de plantas e algas, exsudatos de plantas, goma de sementes e goma microbiana são comumente utilizados em alimentos processados e outros produtos (Medina-Torres, 2000).

As propriedades químicas da mucilagem dos frutos e cladódios de Cereus hildmaniannus K. Schum ainda são desconhecidas, visto que não existem muitos dados científicos na literatura sobre seus usos e viabilidade tecnológica. A maioria dos estudos referese ao gênero Opuntia, que é mundialmente conhecida e consumida. Em alguns países, a mucilagem da Opuntia tem sido utilizada na medicina popular como analgésicos, antibióticos, diuréticos e anti-inflamatórios (Villaseñor et al., 2008). Visando encontrar aplicações tecnológicas também para o $C$. hildmannianus, este trabalho apresenta a caracterização reológica da mucilagem dos frutos e cladódios do Cereus hildmaniannus K. Schum.

\section{MATERIAIS E MÉTODOS}

\subsection{Materiais}

Os frutos e cladódios de Cereus hildmaniannus K. Schum foram coletados em Zortéa, Santa Catarina, Brazil, safra 2010.

Após a recepção, os frutos (Figura 1) foram higienizados com solução de hipoclorito de sódio, lavados com água destilada e cortados para a remoção da polpa. A polpa extraída foi acondicionada em embalagens plásticas e armazenadas a $-18^{\circ} \mathrm{C}$ até utilização.

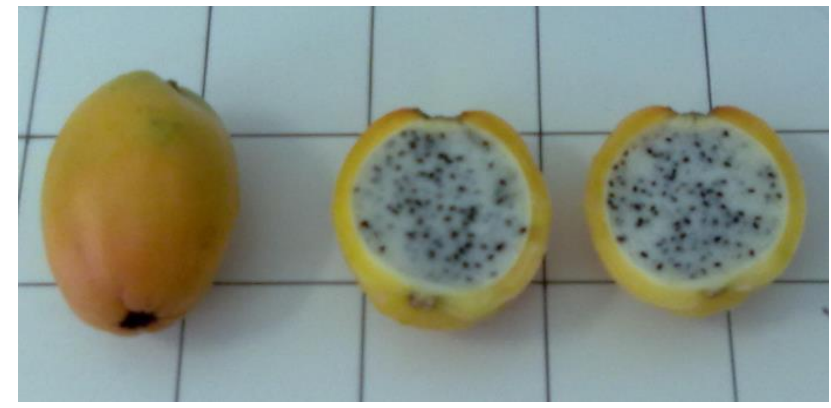

Figura 1 - Fruto do Cereus hildmaniannus lavado e cortado.

Os cladódios, por outro lado, tiveram seus espinhos retirados (Figura 2), sendo posteriormente lavados com água destilada, acondicionados em embalagens plásticas e armazenados em congelador a $-18^{\circ} \mathrm{C}$ até análises posteriores.

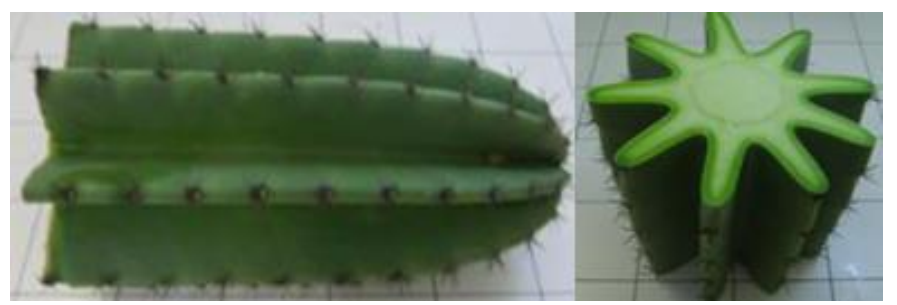

Figura 2 - Cladódio do Cereus hildmaniannus com espinhos e cortado. 


\subsection{Extração da mucilagem}

A polpa dos frutos, descongelada, foi submetida a processo de extração para separação das frações solúvel e insolúvel. Após agitação em agitador mecânico, a polpa foi centrifugada e as duas frações obtidas. Os cladódios descongelados, descascados e cortados foram colocados em um béquer com água para extração a quente da polpa mucilaginosa com auxílio de um agitador mecânico. A amostra foi centrifugada para a separação das frações solúvel e insolúvel. As frações solúveis do fruto e cladódio foram precipitadas com etanol na proporção $3: 1(\mathrm{v} / \mathrm{v})$ e liofilizadas para as análises posteriores. Segundo Iturriaga et al. (2007), o método mais adequado para a precipitação da fração solúvel é o que utiliza etanol, sendo este um método rápido, barato e atóxico quando comparado ao método de precipitação com acetona.

\subsection{Medidas da viscosidade intrínseca}

Soluções aquosas das frações solúveis do fruto e cladódio em concentrações variando de 0,1 a $0,5 \mathrm{~g} \cdot \mathrm{L}^{-1}$ foram preparadas para a análise. A viscosidade das soluções foi medida com viscosímetro capilar, modelo Cannon-Fenske, $\mathrm{n}^{\circ} 75$, acoplado a um banho termostatizado com temperatura ajustada em $30{ }^{\circ} \mathrm{C}$. A viscosidade relativa $\left(\eta_{\text {rel }}\right)$ foi calculada através do tempo de escoamento das soluções e da água pura usando a Equação 1:

$$
\eta_{\text {rel }}=\frac{\eta_{\text {solução }}}{\eta_{\text {solvente }}}
$$

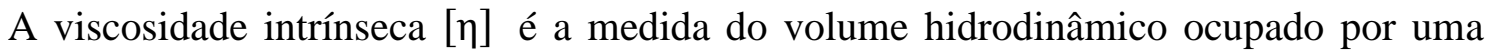
macromolécula. É determinada através de medidas da viscosidade específica de soluções do polímero diluído segundo a Equação 2 (Higiro et al., 2007):

$$
[\eta] \underset{C \rightarrow 0}{=\lim }\left(\frac{\eta_{s p}}{C}\right)
$$

onde $\mathrm{C}$ é a concentração da solução e $\eta_{s p}$ é a viscosidade especifica de acordo com a Equação 3:

$$
\eta_{s p}=\eta_{\text {rel }}-1
$$

A viscosidade intrínseca é geralmente estimada por extrapolação gráfica plotando $\eta_{\mathrm{sp}} / \mathrm{C}$ ou ( $\left.\ln \eta_{\text {rel }}\right) / C$ em função da concentração C, como mostra a Equação 2. Deste modo, é possível correlacionar os mesmos com as equações de Huggins e Kraemer (Equações 4 e 5, respectivamente) (Lai et al., 2000).

$$
\frac{\eta_{s p}}{C}=[\eta]+k^{\prime}[\eta]^{2} C
$$


onde $k^{\prime}$ é a constante de Huggins; e $k^{\prime \prime}$ a constante de Kraemer, respectivamente.

Quanto maior a afinidade entre o polímero e o solvente, maior será o valor da viscosidade intrínseca (Tager, 1972).

A constante de Huggins mede as interações hidrodinâmicas entre as macromoléculas (Lai et al., 2000) e fornece informações sobre as interações entre o polímero e o solvente. Quanto menor for seu valor, melhor deverá ser o solvente, ou seja, mais solvatada estará a macromolécula. Por sua vez, a constante de Kraemer depende da razão entre o volume hidrodinâmico ocupado por duas moléculas de soluto que interagem entre si e o volume hidrodinâmico ocupado por uma molécula isolada.

A concentração crítica de overlap $\left(C^{*}\right)$ é definida como a concentração em que a sobreposição das espirais começa a ocorrer, ou seja, é a concentração de transição das soluções poliméricas do regime diluído para o semi-diluído. Pode ser representada pela Equação 6 (Launay et al., 1997):

$$
C^{*}=\frac{1}{[\eta]}
$$

\section{RESULTADOS E DISCUSSÃO}

As Figuras 3 e 4 apresentam os gráficos de Huggins e Kraemer para a fração solúvel do fruto e cladódio em solução, respectivamente.

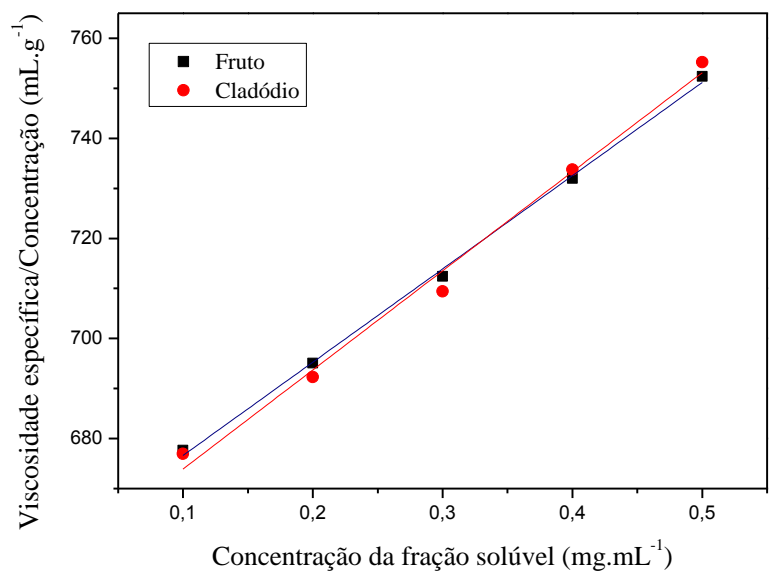

Figura 3 - Gráfico de Huggins da fração solúvel do fruto e cladódio. 


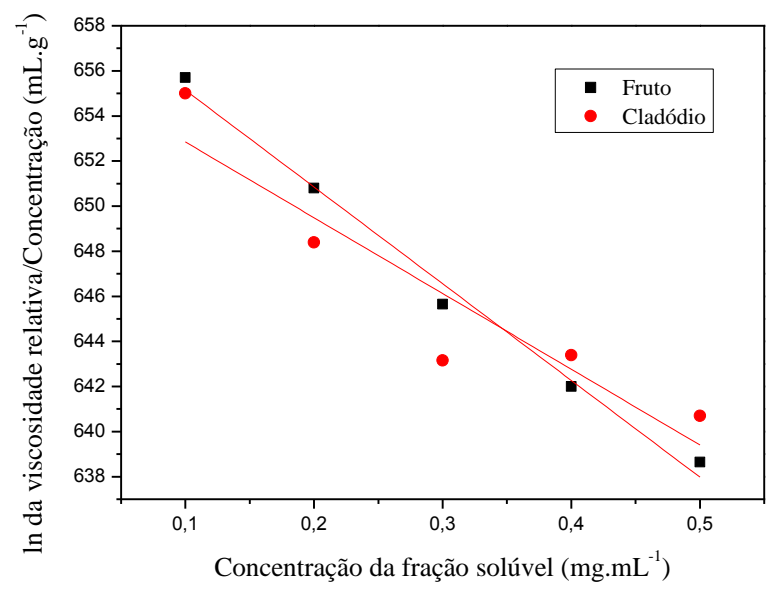

Figura 4 - Gráfico de Kraemer da fração solúvel do fruto e cladódio.

A Tabela 1 mostra os parâmetros obtidos das equações de Huggins e Kraemer para o comportamento da fração solúvel do fruto e cladódio em solução. As viscosidades intrínsecas são representadas pelos coeficientes lineares de cada reta e a concentração crítica de overlap foi calculada pela Equação 6 .

Tabela 1 - Parâmetros das equações de Huggins e Kraemer para as soluções da fração solúvel do fruto e cladódio

\begin{tabular}{|c|c|c|c|c|}
\hline Fração solúvel & \multicolumn{2}{|c|}{ Fruto } & \multicolumn{2}{c|}{ Cladódio } \\
\hline Equação & Huggins & Kraemer & Huggins & Kraemer \\
\hline$[\eta]\left(\mathrm{mL} \mathrm{g}^{-1}\right)$ & 657,99 & 659,43 & 654,11 & 656,21 \\
\hline Constante & 0,43 & $-0,0001$ & 0,46 & $-0,0001$ \\
\hline $\mathrm{C}^{*}\left(\mathrm{~g} \mathrm{~L}^{-1}\right)$ & 1,52 & 1,52 & 1,53 & 1,52 \\
\hline
\end{tabular}

A viscosidade intrínseca, [ $\eta]$, foi menor quando comparada com a mucilagem dos cladódios de Opuntia ficus-indica, que apresentou viscosidade intrínseca entre 850 e 1050 $\mathrm{mL} \cdot \mathrm{g}^{-1}$ para diferentes autores (Majdoub et al., 2001; Goycoolea e Cárdenas, 2003). Wang e Cui (2005) relataram que cadeias mais lineares implicam valores mais elevados de viscosidade intrínseca em função do aumento das interações entre as cadeias de polímero.

Os valores da constante de Huggins obtidos para a fração solúvel do fruto $(0,43)$ e do cladódio $(0,46)$ indicam que a água é um bom solvente para a fração solúvel, nas concentrações estudadas. Esta constante fornece informações sobre as interações entre o solvente e polímero. Valores inferiores a 0,5 indicam que o solvente utilizado é adequado, ou seja, a macromolécula está mais solvatada. A constante de Kraemer $(-0,0001)$ apresenta um valor negativo para as 
duas amostras, significando boa solvatação da cadeia polimérica, isto é, há maior interação polímero-solvente.

As concentrações críticas de overlap foram praticamente iguais para o fruto e cladódio. Sabe-se que acima da concentração crítica de overlap ocorre uma mudança nas propriedades de escoamento devido à formação de entrelaçamentos intermoleculares. Em concentrações mais baixas, as cadeias estão livres para se moverem individualmente, ou seja, as soluções estão em regime diluído ou newtoniano (Mello et al., 2006). A literatura relata que os valores das concentrações críticas de overlap variam muito entre os biopolímeros. Morris et al. (1981) observaram os valores de $0,22 \mathrm{~g} \cdot \mathrm{L}^{-1}$ para goma guar, $1 \mathrm{~g} \cdot \mathrm{L}^{-1}$ para o alginato e $8 \mathrm{~g} \cdot \mathrm{L}^{-1}$ para a dextrana.

\section{CONCLUSÃO}

Os resultados obtidos para a viscosidade intrínseca indicaram boa afinidade entre o polímero e o solvente, e como consequência, as cadeias moleculares encontram-se expandidas no meio líquido. A constante de Huggins demonstrou que a água é um bom solvente para a fração solúvel do fruto e cladódio, enquanto a constante de Kraemer indicou boa solvatação da cadeia polimérica pelo solvente. Acima da concentração crítica de overlap ocorrerá uma interpenetração das cadeias poliméricas, e abaixo desse valor a probabilidade de existir interação entre as macromoléculas em solução é muito baixa.

\section{REFERÊNCIAS}

GERESH, S.; DAWADI, R. P.; ARAD, S. Chemical modifications of biopolymers: quaternization of the extracellular polysaccharide of the red microalga Porphyridium $s p$. Carbohydr. Polym., v. 43, n. 1, p. 75-80, 2000.

GOYCOOLEA, F.; CÁRDENAS, A. Pectins from Opuntia spp.: a short review. J. Prof. Assoc. Cactus Dev., v. 5, p. 17-29, 2003.

HIGIRO, J.; HERALD, T. J.; ALAVI, S.; BEAN, S. Rheological study of xanthan and locust bean gum interaction in dilute solution: Effect of salt. Food Res. Int., v. 40, n. 4, p. 435-447, 2007.

ITURRIAGA, L.; SÁNCHEZ, S.; CORVALÁN, M. Solubility of dispersions of Opuntia ficusindica. Proceedings of the VI International Congress on Cactus Pear and Cochineal, p. 421426, 2007.

JANI, G. K.; SHAH, D. P.; PRAJAPATI, V. D.; JAIN, V. C. Gums and mucilages: versatile excipients for pharmaceutical formulations. Asian J. Pharm. Sci., v. 4, n. 5, 2009.

LAI, L. S.; TUNG, J.; LIN, P. S. Solution properties of hsian-tsao (Mesona procumbens Hemsl) leaf gum. Food Hydrocolloids, v. 14, n. 4, p. 287-294, 2000. 
LAUNAY, B.; CUVELIER, G.; MARTINEZ-REYES, S. Viscosity of locust bean, guar and xanthan gum solutions in the Newtonian domain: a critical examination of the log ([eta] sp) o$\log$ c [[eta]] o master curves. Carbohydrate Polymers, v. 34, n. 4, p. 385-395, 1997.

MAJDOUB, H.; ROUDESLI, S.; PICTON, L.; LE CERF, D.; MULLER, G.; GRISEL, M. Prickly pear nopals pectin from Opuntia ficus-indica physico-chemical study in dilute and semi-dilute solutions. Carbohydr. Polym., v. 46, n. 1, p. 69-79, 2001.

MEDINA-TORRES, L.; BRITO-DE LA FUENTE, E.; TORRESTIANA-SANCHEZ, B.; KATTHAIN, R. Rheological properties of the mucilage gum (Opuntia ficus-indica). Food Hydrocolloids, v. 14, n. 5, p. 417-424, 2000.

MELlO, I. L.; COUTINHO, F. M. B.; DELPECH, M. C.; ALBINO, F. F. M.; SANTOS, S. M. Polibutadieno alto-cis: estudo viscosimétrico em tolueno e ciclo-hexano. Polímeros Ciência e Tecnologia, v. 16, n. 1, p. 53, 2006.

MORRIS, E. R.; CUTLER, A. N.; ROSS-MURPHY, S. B.; REES, D. A.; PRICE, J. Concentration and shear rate dependence of viscosity in random coil polysaccharide solutions. Carbohydr. Polym., v. 1, n. 1, p. 5-21, 1981.

PERIAGO, M. J.; ROS, G.; LÓPEZ, G.; MARTINEZ, M. C.; RINCÓN, F. The dietary fiber components and their physiological effects. Revista Española de Ciencia y Tecnología de Alimentos, v. 33, n. 3, 1993.

SÁENZ, C.; SEPÚLVEDA, E.; MATSUHIRO, B. Opuntia spp mucilage's: a functional component with industrial perspectives. J. Arid Environ., v. 57, n. 3, p. 275-290, 2004.

TAGER, A. Physical Chemistry of Polymers: Moscow: Mir Publishers, 1972.

VILLASEÑOR, M. A. A.; BOIX, A. C.; NAVARRETE, N. M. Efecto del método de extracción en las características químicas y físicas del mucilago del nopal (Opuntia ficus-indica) y estudio de su aplicación como recubrimiento comestible. 2008. (Doutorado). Departamento de Tecnología de Alimentos, Universidad Politécnica de Valencia, Valencia.

WANG, Q.; CUI, S. W. Understanding the physical properties of food polysaccharides. Food Carbohydrates: Chemistry, Phisical Properties and Applications, 2005. 